\title{
LAS FUENTES DE LA HISTORIA DE HERODIANO
}

\begin{abstract}
Without doubt the most important and most debated subject of the sources utilized by Herodian is the use he made of Cassius Dio. Even considering that the subject may be open to discussion, it would be difficult to defend the argument that Herodian used Cassius Dio exclusively or basically as a historical source even when he wrote on the same topics that Cassius Dio did. There is, however, a general agreement on evaluating more highly the last part of Herodian's work.
\end{abstract}

Los mediadores entre los sucesos que se narran y la propia narración histórica ya eran considerados por la preceptiva historiográfica de la antiguiedad temas de la mayor importancia '. Por ello es frecuente ver cómo los historiadores recomendaban la veracidad de sus obras por la contemporaneidad de ellos con los sucesos que se narraban en las mismas $\mathrm{y}$, a ser posible, por su condición de testigos cualificados ${ }^{2}$.

No debe, pues, sorprender que Herodiano desde el mismo prólogo de su Historia, un lugar idóneo para hacer una declaración de principios, diga:

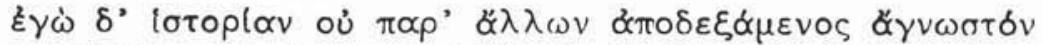

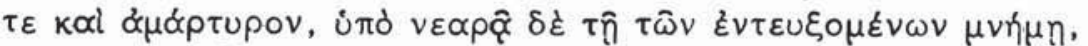

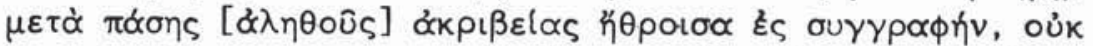

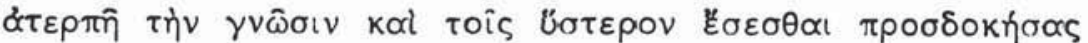

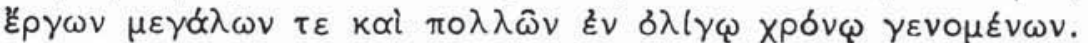
$(\mathrm{I} 1,3)$

1 Hasta el punto de que según algunos autores servían para definir un género. Aulo Gelio, NA V 18, citando a Verrio Flaco, decía que la palabra estaba limitada a designar narraciones de sucesos contemporáneos. C. R. Whittaker, en su ed. de Herodiano, Londres-Cambridge (Mass.) $1969-70$, vol. I, p. 4, n. 1, indica que la palabra historia $(\mathrm{I} 1,3)$ quizá pudo ser utilizada por Herodiano en este sentido preciso.

2 Ello es evidente, por ejemplo, en un historiador casi contemporáneo de Herodiano: el senador Casio Dión. En un pasaje justifica la introducción de cierto tipo de noticias por su condición de contemporáneo y por su deseo de narrar estos sucesos coetáneos con mayor cuidado que los precedentes (LXXII 18, 3-4). 
Sin embargo, esta rotunda afirmación debe ser matizada y de hecho lo ha sido por diversos estudiosos ${ }^{3}$. Además, el mismo Herodiano da noticias de distintas fuentes, algunas de las cuales, las menos, son designadas, y otras, las más numerosas, según es habitual entre los historiadores de la antigüedad, quedan encubiertas por fórmulas impersonales. Pasemos, pues, revista a los datos que el propio historiador nos ofrece sobre su obra. Habla de una serie de escritos y discursos que legó Marco Aurelio (I 2, 3) ${ }^{4}$ y, además, menciona la Autobiografía de Septimio Severo (II 9, 4) ${ }^{5}$. Se refiere también Herodiano a distintas obras, aunque sin nombrar a los autores, que se ocuparon en especial de las actividades bélicas de estos emperadores. Así menciona a los

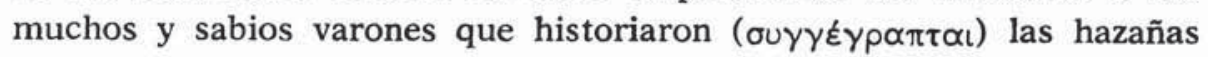
realizadas con talento político y militar en el Norte y el Este por Marco Aurelio (I 2, 5) ${ }^{6}$. Asimismo dedica un comentario a los autores, historiadores y poetas que se ocuparon de las campañas de Septimio Severo durante la guerra civil, de las fases de su avance y de los discursos pronunciados en las ciudades, del número de bajas de los distintos bandos y de los signos atribuidos a la providencia divina (II 15, 6) ?

3 Piénsese que la Historia de Herodiano cubre desde el año 180 al 238, y los primeros años de este período corresponderían a una fase muy temprana en la vida de Herodiano en la que difícilmente podía tener una cumplida conciencia de lo que sucedía a su alrededor. Cf. F. Cassola "Sulla vita e sulla personalità dello storico Erodiano», NRS 41, 1957, pp. 216-8, y G. Alföldy "Herodian's Person», AncSoc 2, 1971, pp. 205-8, sobre las fechas que sugieren sobre la publicación de la obra de Herodiano y en su caso sobre la fecha de nacimiento, etc.

4 No es seguro que se refiera a las meditaciones, cf. Whittaker, op. cit., vol. I, p. 9, n. 5 .

5 La Autobiografía de Septimio Severo es mencionada, además, por Casio Dión, LXXV 7, 3; A. Víctor, de Caes. XX 22 y en SHA Seu. III 2; Nig. IV 7; Alb. VII 1 y X 1 . Sobre la fecha en la que quizá fue escrita (205) y los posibles colaboradores según estuviera en latín o griego (Mesio Saturnino y Elio Antípatro), cf. A. Birley, Septimius Severus. The African Emperor, Londres 1971, p. 240 ss.

6 Cf. Whittaker, op. cit., vol. I, p. 10, n. 2.

7 Vuelve a mencionar a algunos que narraron la guerra civil, en concreto la campaña contra Albino en III 7, 3 y 6 . Sobre los autores que se ocuparon de estas cuestiones podemos mencionar en primer lugar a Casio Dión, quien nos dice que en primer lugar escribió un pequeño libro sobre los sueños y signos por los que Severo esperaba ser emperador y que, tras tener noticia de la buena acogida de su obra ante el emperador, se determinó a escribir una segunda obra que comenzando en los últimos momentos de Cómodo se ocupaba de las revueltas y guerras que tuvieron lugar después de su muerte (LXXII 23. Cf. también LXXIII 14, 4 y LXIV 8, 2). Sobre la fecha de redacción de estas obras, etc., cf. F. Gascó, La concepción historiografica de Casio Dión, tesis mecanografiada, Sevilla 1981, p. 25 ss., y C. Letta, ${ }^{2}$ La composizione dell'opera di Cassio Dione: cronologia e sfondo storico-politico», en Ricerche di storiografia Greca di età Romana, Pisa 1979, pp. 117-189. 
Fuera de estas indicaciones que poseen alguna precisión, sólo contamos con referencias a obras anónimas y autores que quedan enmascarados en fórmulas muy poco explícitas por las que simplemente se indica que un contenido semejante se puede encontrar en otros autores

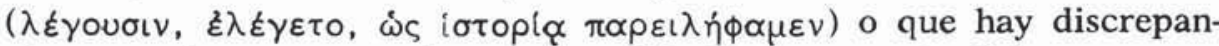

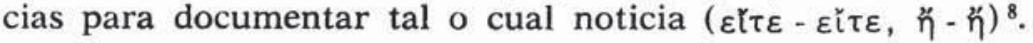

De estas referencias las que poseen un menor interés histórico son las relativas a temas de carácter erudito, en las que los usos religiosos ocupan un lugar destacado 9 . Estas digresiones menudean en los dos primeros libros y deben conectarse con un hábito de la segunda sofística, moda con la que sin duda Herodiano quería acrecer el interés de su narración. Por constituir estas digresiones un elemento habitual en la literatura de la época ${ }^{10}$ se deben considerar acertadas las reservas de Whittaker ${ }^{11}$ para coincidir con la opinión de Baaz ${ }^{12}$, acogida por Dopp ${ }^{13}$, según la cual esta información la habría obtenido Herodiano de Verrio Flaco, autor cuya obra habría sido resumida durante el s. II. La obra de este autor o su epítome constituirían tan sólo dos de entre las múltiples posibilidades a las que Herodiano pudo recurrir.

Sin duda el aspecto de Herodiano que ha suscitado un más vivo interés entre los estudiosos ha sido el del uso que hizo de la Historia de Roma de Casio Dión al escribir su obra. El tema ha sido amplia y largamente debatido y ello debido a distintas circunstancias. Por una parte, está el estado fragmentario o resumido de los últimos libros de la Historia de Roma de Casio Dión ${ }^{14}$; por otra, el silencio de Herodiano sobre sus fuentes al que ya hemos aludido ${ }^{15}$; $\mathrm{y}$, por fin, el hecho de que no conservemos alguna de las obras que menciona relativas a la

8 La mejor sistematización de estos pasajes, tras la que ya en su día realizó E. Baaz, De Herodiani fontibus et auctoritate, Diss. Berlín 1909, pp. 6-15, es la realizada por Whittaker, $o p$. cit., vol. I, pp. LXI-LXIII: estas citas se encuentran a) de forma constante en conexión con información anticuaria; b) frecuentemente cn conexión con sucesos contemporáneos; c) a veces en relación con noticias geográficas o historiográficas; d) a veces indican más de una fuente.

9 "Qua in disquisitione primum intelegimus Herodianum libenter de rebus ad ritum religionis pertinentibus agere ibique semper confiteri se ea ab aliis accepissen, Baaz, op. cit., p. 7.

10 B. P. Reardon, Courants littéraires grecs des II e et IIIe siècles après J. C., París 1971, p. 206 ss.

11 Op. cit., vol. I, p. LXII.

12 Op. cit., pp. 7-13.

13 RE VIII, col. 956.

14 F. Millar, A Study of Cassius Dio, Oxford 1964, pp. 1-4.

15 Los estudiosos de Herodiano recuerdan lo acertado de las palabras de Mendelssohn en su edición de este autor (Herodiani ab excessu Divi Marci libri octo, Leipzig 1883, p. XIX), cf. Baaz, op. cit., p. 7, y Dopp, RE VIII, col. 958. 
vida y campañas de Septimio Severo, que nos podrían dar pistas importantes sobre la forma de trabajo de Herodiano ${ }^{16}$.

Cuando Baaz, en 1909, en el primer capitulo de su obra ofrece las razones por las que consideraba necesario un trabajo sobre las fuentes de Herodiano, en especial en su relación con Casio Dión, se refirió a la disparidad de opiniones existentes en torno al tema ${ }^{17}$. Creemos significativo que Widmer en la primera página del prólogo de su obra sobre Herodiano, publicada en 1967, dijera que todavía se estaba por alcanzar una solución satisfactoria sobre el tema de las fuentes de Herodiano ${ }^{18}$ y se limitaba, en lo que respecta a las relaciones con Casio Dión, a citar las múltiples opiniones existentes, que por cierto cubren todas las posibilidades imaginables ${ }^{19}$.

En este estado de la cuestión, poco alterado por las prudentes páginas que Whittaker dedicó al tema ${ }^{20}$, se debe situar el trabajo de Kolb ${ }^{21}$. $\mathrm{Su}$ intención fue obtener alguna solución al problema de las fuentes de la Historia Augusta a partir no de autores y obras perdidas o de dudosa identidad, sino a partir de autores y obras que se conservan y cuya utilización por la Historia Augusta es declarada ${ }^{22}$. Mientras cumple este objetivo, se convierte también en necesario objeto de consideración de este autor la discutida relación entre Herodiano y Casio Dión ${ }^{23}$. Tras un estudio detallado de distintos sucesos narrados por Casio Dión y Herodiano ${ }^{24}$ el resultado al que llega Kolb, en lo que respecta a la relación existente entre las obras de ambos autores, es que la obra de Herodiano constituye una historia novelada con superficiales juicios morales cuya fuente más importante es la Historia de Roma de Casio Dión. Añade a esto que las divergencias entre una y

16 Cf. W. Widmer, Kaisertum, Rom und Welt in Herodians META MAPKON

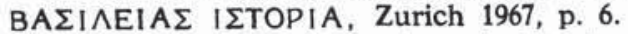

${ }_{17}$ Cita los puntos de vista de Volckmann, Poblocki, Höfner, Kreutzer, Mendelssohn, Wirth, Thiele, Schwartz y Niese en op. cit., pp. 5-6.

18 «ine befriedigend Lösung der Quellenfrage ist noch immer aussichtslos...», op. cit., p. 5.

19 Op. cit., p. 6, n. 7.

20 Op. cit., pp. LXI-LXXI.

21 Literarische Beziehungen zwischen Cassius Dio, Herodian und der Historia Augusta, Bonn 1972. Antes de que apareciera publicado, las conclusiones de este trabajo fueron citadas y utilizadas para aquellos aspectos que afectan a temas relacionados con la biografía de Herodiano por G. Alföldy, op. cit., pp. 206 y 231.

2 «Daher durfte es ergiebiger sein, das...n, op. cit., p. 4.

23 Op. cit., pp. 6-7.

24 Los temas son: Cómodo en el Anfiteatro, la conspiración contra Cómodo, la renuncia de Pompeyano al Imperio, el ascenso at trono de D. Juliano, la batalla en las «Puertas Cilicias» entre S. Severo y P. Níger y la digresión sobre la ciudad de Bizancio, la matanza de alejandrinos por Caracala, la campaña de Caracala contra los partos, la muerte de Caracala y la guerra pártica de Macrino. 
otra obra se explicarian, según Kolb, por ampliaciones retóricas que se hallan en profunda contradicción con las grandilocuentes frases del prólogo de la obra de Herodiano 25 .

Sin embargo, resulta significativo el eco que ha producido la obra de Kolb entre estudiosos todos ellos buenos conocedores del autor y su época. Mientras que G. Alföldy ${ }^{26}$ y A. Birley ${ }^{27}$ aceptan las conclusiones del trabajo de Kolb, Cassola por su parte ${ }^{28}$ considera, en primer lugar, que ninguno de los ejemplos aportados por Kolb conduce a una conclusión tan evidente como pretende $\mathrm{y}$, en segundo lugar, que en la obra de Kolb queda por explicar por qué Herodiano se tiene que remitir de forma constante y sistemática a una obra que narra sucesos que también son contemporáneos del propio Herodiano y de los que, por tanto, podía tener información y opiniones que no pasaran por el circuito dioneo. A esta autorizada opinión se debe añadir la importante reseña que la obra de Kolb recibió de mano de Barnes ${ }^{29}$. Según este estudioso, las conclusiones de Kolb son discutibles fundamentalmente porque, si de forma sistemática se explican las divergencias entre Casio Dión y Herodiano por transposiciones o transferencias de pasajes, ampliaciones retóricas o alteraciones a voluntad, se cierra el paso de forma arbitraria a toda explicación que intente justificar las divergencias partiendo de una base documental diferente, y esto es tanto más infundado cuanto que se conoce la existencia de obras que efectivamente pudieron ser utilizadas ${ }^{30}$.

Un breve pero importante trabajo de Bowersock ${ }^{31}$ insistía en la dirección indicada por Cassola y Barnes. Señalaba cuatro casos en la narración sobre Heliogábalo ${ }^{32}$ en los que la Historia de Herodiano está mejor informada que la de Casio Dión.

Las discrepancias existentes para los libros de Herodiano que narran los mismos sucesos que son tratados por la Historia de Roma de Casio Dión disminuyen para los últimos libros. Es opinión generalizada que los defectos de Herodiano, sino desaparecen, sí al menos se atenúan cuando llega su obra al ascenso de Maximino y a la revuelta de los

25 Op. cit., pp. $160-1$.

26 Op. cit., pp. 206 y 231.

27 JRS 64, 1974, pp. 266-8.

28 Athenaeum 52, 1974, pp. 374-8.

29 Gnomon 47, 1975, pp. 368-373. Reitera esta misma opinión, aunque añadiendo el trabajo de Bowersock que citaremos a continuación, en su The Sources of the Historia Augusta, Bruselas 1978, pp. 79-89.

30 Casio Dión, Antipatro, Mario Máximo y Asinio Cuadrato.

31 «Herodian and Elagabalus», en D. Kagan (ed.). Studies in the Greek Histo. rians, Cambridge (Mass.) 1975, pp. 229-236.

${ }_{32}$ Los pasajes que estudia son: V 3,$3 ; \mathrm{V} 3,5 ; \mathrm{V} 5,6 ; \mathrm{V} 6,3$. 
Gordianos ${ }^{33}$. En gran medida entendemos que es una opinión inconfrontable, puesto que la Historia Augusta utiliza para las biografías de Maximino y los Gordianos a Herodiano y la crítica externa no siempre es posible. A pesar de ello, y añadiéndose a la proximidad temporal de estos sucesos a la ejecución de la Historia, hay en la propia narración de Herodiano algunos indicios que podrían indicar la utilización de fuentes bien informadas ${ }^{34}$. Todo ello no impide que se mantegan reservas fundadas también en estos libros por el talante retórico del autor ${ }^{35}$, por la falta de precisión de su narración ${ }^{36}$ y por su propio punto de vista político ${ }^{37}$.

FERnANDo Gascó

33 A pesar de sus generalizaciones y retórica la valoración de Maximino por Herodiano le parece aceptable en bastantes aspectos a G. M. Bersanetti (Studi sull'imperatore Massimino il Trace, Roma 1940, p. 73 ss.). Rostovzeff consideraba los libros de Herodiano ocupados con los sucesos protagonizados por Maximino y los Gordianos "una pintura admirable» de la época (Historia social y cconómica del I. romano, Madrid 1937, vol. II, pp. 327,333 y 380, n. 31). También según Cassola la parte mejor de su obra está constituida por los dos últimos libros, en los que narra con viveza la revuelta contra Maximino y el ascenso de los Gordianos (cf. op. cit., p. 221). Más reciente Gagé («Les organizations de iuvenes en Italia et en Afrique du début du III se siècle au bellum Aquileiense», Historia 19, 1970, p. 323 ss.) encuentra un fondo de verdad en las arengas que Herodiano pone en boca de Maximino y una inscripción hallada en Aquilea concuerda con la narración de Herodiano. Loriot (ANRW II 2, 1975, pp. 660-1) entiende que es una fuente irreemplazable, y que los sucesos de los años 235-8 impresionaron vivamente al autor, y que sus defectos como historiador se atenúan para estos momentos. Dietz (Senatus contra principem, Munich 1980, pp. 33-6) considera que la opinión según la cual los últimos libros de Herodiano son superiores a los anteriores no es infundada, aunque ello no quiere decir que haya que aceptar de forma irreflexiva toda su narración.

34 Whittaker, op. cit., vol. I, pp. XXXI-V, esp. XXXIII. Según este estudioso Herodiano estaría en Roma y habría podido utilizar fuentes de primera mano; sin embargo, cf. Alföldy, op. cit., pp. 230-3. 185.

35 Acentúa este aspecto R. Syme, Emperors and Biography, Oxford 1971, pp. 184 -

36 K. Dändliker, «Die drei letzen Bücher Herodians», en M. Bündiger (ed.), Untersuchungen zur römischen Kaisergeschichte, vol. III, Leipzig 1870, pp. 240-1.

37 Widmer, op. cit., pp. 18 s., 22,25 s., 31 s., 44. 